\title{
Prenatal Ultrasound Diagnosis of Fetal Urogenital Anomalies
}

\author{
${ }^{1}$ Ana Tikvica Luetic, ${ }^{2}$ Asim Kurjak
}

\begin{abstract}
Congenital urogenital anomalies constitute approximately 20 to $30 \%$ of all malformations identified in the antenatal period. Since fetal urogenital malformations are a significant cause of neonatal and child morbidity and mortality, the necessity of providing the correct diagnosis for the parents and professionals involved in the management should be of great priority.

Here, we present a review of the most common fetal urogenital malformations with special consideration of ultrasound features and addition of our ultrasound assessments.
\end{abstract}

Keywords: Autosomal dominant polycystic kidney disease, Urogenital anomalies, Ultrasound diagnosis.

How to cite this article: Luetic AT, KurjakA. Prenatal Ultrasound Diagnosis of Fetal Urogenital Anomalies. Donald School J Ultrasound Obstet Gynecol 2016;10(3):301-307.

Source of support: Nil

Conflict of interest: None

\section{INTRODUCTION}

The prevalence of fetal malformation is relatively high, with an average prevalence of $2.0 \% .{ }^{1}$ Among all the malformations, anomalies of the urogenital tract seem to be among the most common, with a prevalence rate of 0.5 to $0.8 \%$ in unselected population according to the literature. ${ }^{2,3}$ If certain organs are considered, then kidney is the most affected site of congenital abnormalities. Since most of the parts of urogenital system can be visualized by ultrasound as early as 12 weeks of gestation and with more confidence later through pregnancy, these anomalies constitute approximately 20 to $30 \%$ of all malformations detected in the antenatal period. ${ }^{4}$

Urogenital malformations can be unilateral or bilateral, mild, and asymptomatic, while in some cases can result in impaired renal function and be lethal in prenatal or postnatal period.

\footnotetext{
${ }^{1}$ Assistant Professor, ${ }^{2}$ Professor and President

${ }^{1}$ Department of Obstetrics and Gynecology, Clinical Hospital Sveti Duh, Zagreb, Croatia

2Department of Obstetrics and Gynecology, International Academy of Perinatal Medicine, Zagreb, Croatia

Corresponding Author: Ana Tikvica Luetic, Assistant Professor Department of Obstetrics and Gynecology, Clinical Hospital Sveti Duh, Zagreb, Croatia, e-mail: ana_tikvica@yahoo.com
}

Additionally, these disorders predispose an individual to many other complications, such as ureteral obstruction, stone formation, infection, hypertension, renal failure, and cardiovascular disease throughout life. Although congenital anomalies of the urinary and genital tracts are a part of many known syndromes, nonsyndromic cases are probably caused by single-gene defects and are more common in families with previously affected member. ${ }^{5}$ Since these congenital disorders are responsible for 30 to $50 \%$ of cases of end-stage renal disease in children, ${ }^{6}$ it is of crucial importance to diagnose these anomalies in order to initiate therapy as soon as possible to minimize renal damage and to prevent or delay the onset of kidney failure. In case of lethal form of congenital kidney disease, it is important to diagnose it in order to give accurate information to the parents and to provide them with proper genetic counseling.

Although prenatal genetic and molecular testing of the different fetal and placental tissues has enabled a huge step-up in antenatal diagnosis of different anomalies of urogenital tract, ultrasound still remains an irreplaceable tool in initial assessment of fetal morphology. With the development of new ultrasound technologies, such as high-definition ultrasound, the diagnosis of different urogenital anomalies has moved from the second to the first trimester, enabling faster diagnosis and the possibility to perform invasive procedures earlier in pregnancy if necessary. The finding of different urogenital anomalies should be followed by detailed examination of entire fetal anatomy and evaluation of the amniotic fluid level. The differential diagnosis should take into account personal and family history, the presence of associated anomalies, karyotype, and genetic testing if available.

Here, we present a review of the use of ultrasound in the detection of fetal urogenital system with special consideration in kidney malformations since it is the most common site of different developmental disruptions.

\section{ANOMALIES OF THE FETAL KIDNEY}

Human kidney develops in early embryonic stage and it evolves through three developmental stages: Pronephros, mesonephros, and metanephros. The earliest stage is the pronephros that develops from the 4 th to the 14 th somites and consists of 6 to 10 pairs of tubules. ${ }^{7}$ These tubules join into a pair of primary ducts that grow caudally, and eventually terminate into the cloaca. The pronephros is a temporary structure that disappears completely by the 
4th week of human embryonic life. Second stage of renal development is mesonephros that derives from the intermediate mesoderm and becomes the principal excretory organ by the 8th embryonic week. Although mesonephros gradually degenerates, the parts of its duct system constitute some parts of the male reproductive organs. The final stage of the renal formation is the metanephros that arises distal to the mesonephros at 5 weeks of development. It is also derived from the intermediate mesoderm. The precursor of the ureters grows as a diverticulum from the Wollfian duct and extends toward and inside the metanephric mesenchyme. ${ }^{7}$

Development of the new ultrasound technologies and improvement of the resolution of the ultrasound picture enabled the earlier visualization of fetal kidneys even at the end of the first trimester. In most of the cases, kidneys can be assessed from the 9 th week of gestation, while the period beyond 12 weeks should provide us with the clear demonstration. During detailed morphologic examination at the 20 gestational weeks in 95\% of fetuses' kidneys should be assessed by ultrasound in detail and analyzed in their number, position, measurements, echogenic appearance. ${ }^{8}$ An indirect sign of renal appearance is the presence or absence of renal arteries that could be examined by color Doppler flow imaging, while the evaluation of the amniotic fluid index gives additional information about the function of the kidney. In normal circumstances ureters are not visible, while their ultrasound appearance should be followed by the detailed examination of the rest of the urogenital and other system anatomy.

The most common renal abnormalities diagnosed prenatally by ultrasound are hydronephrosis caused by transient urine flow impairment at the level of the pelvis-ureteric junction and vesico-ureteric junction or posterior urethral valve. ${ }^{9}$ In most cases, hydronephrosis is diagnosed in the absence of other urogenital anomalies as the dilatation of anterior-posterior pelvis diameter above the referral values for certain gestational age. This is often considered as physiologic changes in male fetuses with good prognosis and spontaneous resolution after birth, while the finding of hydronephrosis in female fetuses carries the higher risk for adverse outcome. ${ }^{10}$ Renal pelvis diameter is a measurement of collecting system dilatation and does not reflect the parenchymal changes of the kidney, such as increased echogenicity, thinning, or caliectasis. The most widely used cut-off value for the pelvis diameter is 4 and $5 \mathrm{~mm}$ in the second trimester, while increase of the values up to $10 \mathrm{~mm}$ is considered to be mild with spontaneous postnatal resolution. On the other hand, values of diameter above $10 \mathrm{~mm}$ are associated with higher risk of congenital anomalies of the kidney and urinary tract with consecutive increase of the risk with the enlargement of the anterior-posterior diameter value. ${ }^{11}$ In order to better predict postnatal complications of the ultrasound diagnosis of antenatal hydronephrosis, the antenatal Society of Fetal Urology developed criteria for the diagnosis and grading of hydronephrosis based upon the degree of pelvic dilatation, number of dilatated calyces, and the presence and severity of parenchyma atrophy. ${ }^{12}$ According to that grading system, grade I includes only mild dilatation of the renal pelvis, while moderate dilatation of the renal pelvis including a few calyces is considered as grade II. Grade III includes uniform dilatation of all calyces with normal renal parenchyma, while grade IV is the most serious with additional thinning of the renal parenchyma (Fig. 1). The prognosis is worse with higher grade with increase number of surgical interventions in grade III and IV. Although mild hydronephrosis, which is usually called pyelectasia, resolves after birth in most cases, their finding should also be followed by the detailed ultrasound assessment of the fetal anatomy and review of clinical risk factors for trisomy 21 since it has been shown that its prevalence is higher in a case of Down syndrome. ${ }^{13}$ Despite this, isolated finding of the pyelectasia is not obligatory for genetic amniocentesis since the risk for Down syndrome remains low in the absence of maternal risk factors and other sonographic markers. ${ }^{13}$

After hydronephrosis, which can be found in up to $1 \%$ of all pregnancies, congenital anomalies of the kidney and urinary tract constitutes a second group of frequent pathology usually found during antenatal diagnostic ultrasound examination. As previously described, the development of the kidney and other parts of the urogenital system in humans is a pretty complicated process, which, on disruption at any time, can cause several different phenotypes with different prognosis. Due to the multiple etiologies of renal anomalies and their similar appearance on ultrasound examination, it is often difficult to establish correct diagnosis during antenatal examination.

Autosomal dominant polycystic kidney disease (ADPKD) is one of the inherited causes of renal disease with the highest prevalence. It is an autosomal dominant condition with high penetration, which can be present in three different forms. It is related to the mutations of different genes that result not only in disturbed tubular and vascular development of the kidneys but also in other organs including the liver, brain, and pancreas. This condition affects about 1 in 1,000 individuals and it accounts for about $10 \%$ of people on dialysis. ${ }^{14}$ Many of the patients have complications of the disease, such as hypertension, impaired urine concentration, hematuria, renal stones, or failure, but there is also a growing number of individuals detected in screening procedures of the relatives. Although ultrasound examination is widely used for kidney evaluation in ADPKD, 


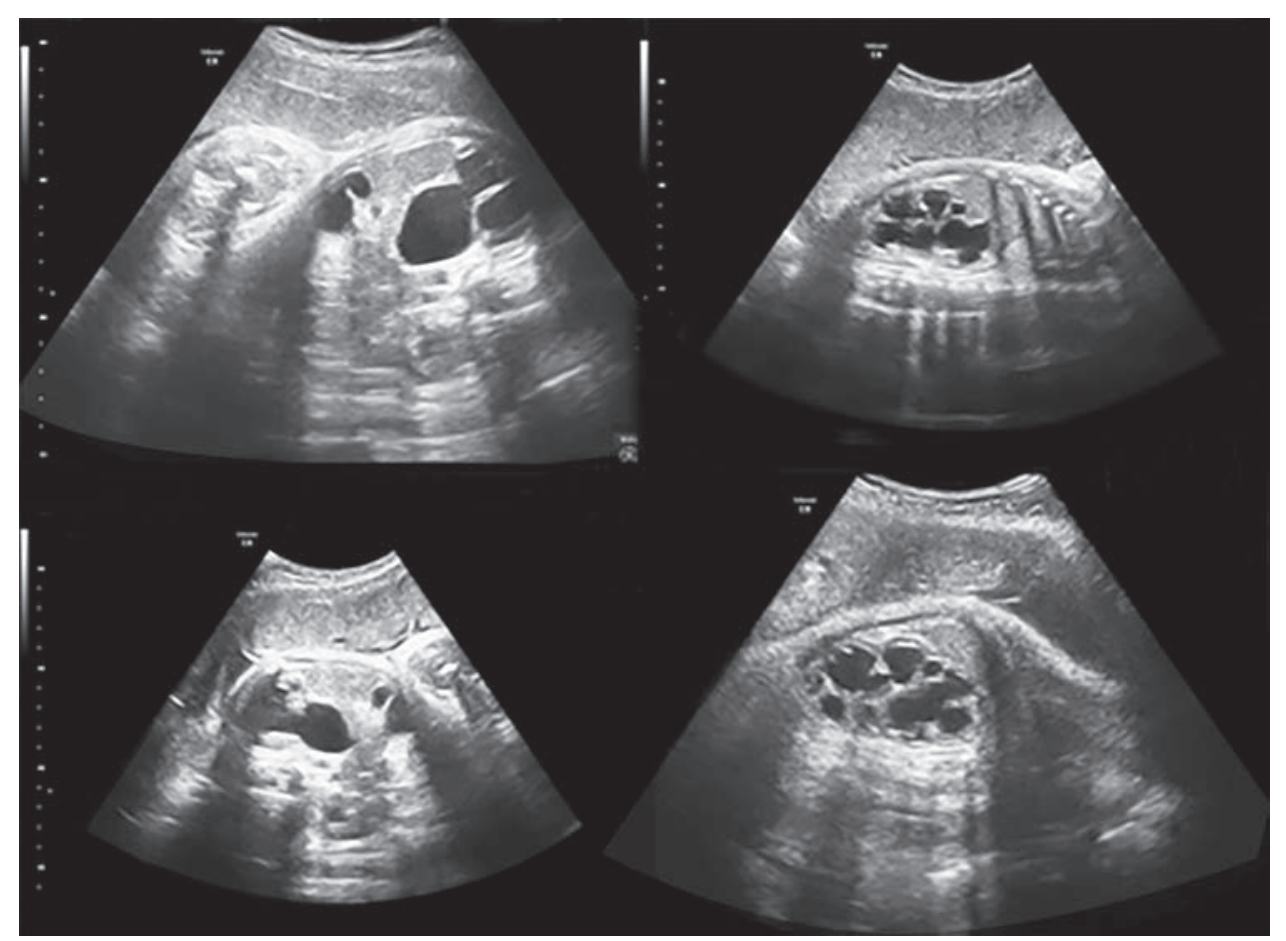

Fig. 1: An ultrasound presentation of hydronephrosis grade IV in the third trimester (severe dilatation of renal pelvis with uniform dilatation of all calyces with thinning of the renal parenchyma)

it has low sensitivity for individuals younger than 30 years. ${ }^{14}$ With the technological improvement of ultrasound, the prenatal diagnosis of ADPKD is now being reported with increasing frequency. Main sonographic findings of the condition are moderate renal enlargement with hyperechogenic renal cortex and hypoechogenic medulla resulting in increased corticomedullary differentiation, while the detection of renal cysts is uncommon (Fig. 2). ${ }^{15}$ By definition, a hyperechogenic kidney is recognized as having a renal cortex more echogenic than the liver and spleen. In a case of ADPKD, amniotic fluid index is usually normal. All described ultrasound signs are not to be missed in a case of affected families; however, the signs can be neglected in a case of unexpected diagnosis. ${ }^{16}$

Autosomal recessive polycystic kidney disease (ARPKD) is a rare autosomal recessive disorder caused by the mutation of the six chromosomes. ${ }^{17}$ In ARPKD, kidneys are usually bilaterally enlarged with many cysts due to the dilatation and elongation of renal collecting ducts. The severity of renal malformation and associated liver disease could be variable even in the same family. ${ }^{17}$ The onset of the ARPKD also varies, which represents the main criteria for the classification of ARPKD; prenatal, neonatal, infantile, and childhood form. Earlier onset of the disorder is associated with serious complications and outcome.

Prenatal ultrasound examination is a very useful imaging tool for the diagnosis of ARPKD with over $50 \%$ of cases being diagnosed prenatally. ${ }^{17}$ Although large kidneys may be visualized from 13 weeks onward,

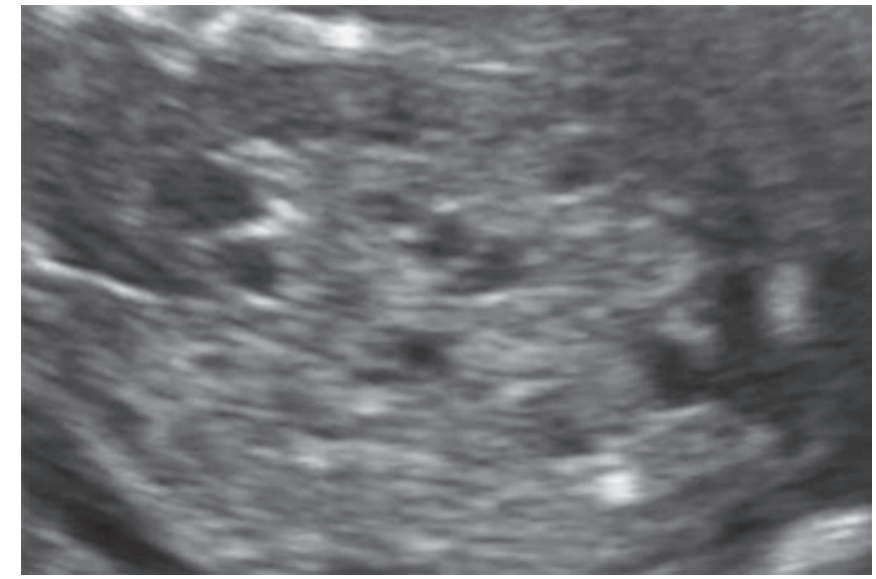

Fig. 2: An ultrasound presentation of moderately enlarged, echogenic kidney in a fetus with ADPKD

the hyperechogenicity of the kidneys that results from the presence of multiple micro cysts, dysplasia, or tubular dilatation should not be missed after 17 weeks' gestation (Fig. 3). ${ }^{18}$ There is also an absence of corticomedullary differentiation within the kidney with decrease in amniotic fluid amount, fetal ascites, and difficulty in demonstration of fetal bladder. ${ }^{19}$ In a case of early onset of the disease, impaired renal function leads to oligohydramnios and consequently to pulmonary hypoplasia, club foot, and Potters' faces. ${ }^{20}$ The prognosis of ARPKD is poor, with death occurring within the first two postnatal months due to the renal or respiratory failure.

Multicystic dysplastic kidney is the most frequent type of renal cystic disease, and it is one of the most common causes of an abdominal mass in infants. Multicystic 


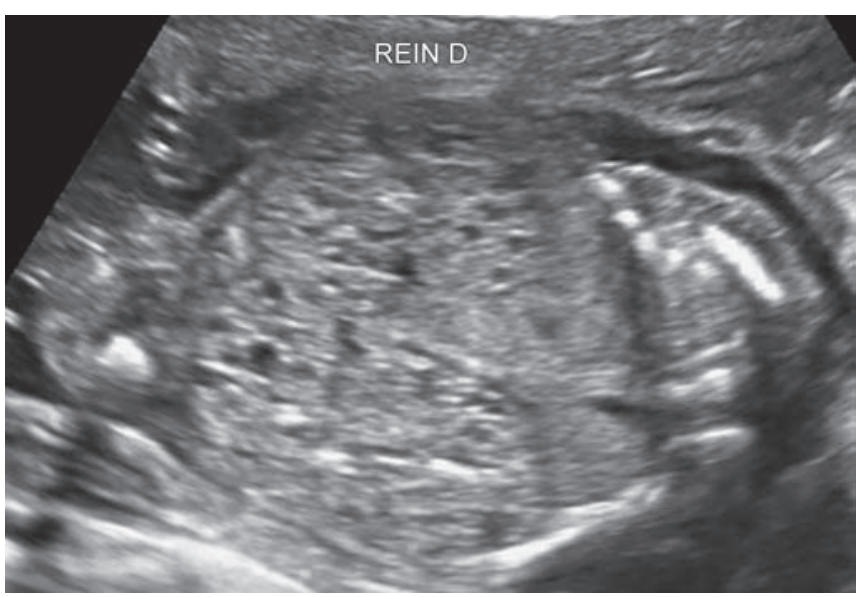

Fig. 3: Ultrasound examination in the second trimester revealing enlarged abdomen with bilaterally symmetrically enlarged echogenic kidneys filling the fetal abdomen in a fetus with ARPKD

dysplastic kidney develops as an architectural disorganization due to the early obstructive defect of the branching tubules in the developing kidney. In most of the cases, this is a unilateral condition with a nonfunctional affected organ, while bilateral condition can be found in $20 \%$ of cases. ${ }^{21}$ About $40 \%$ of fetuses with multicystic dysplastic kidney have additional genitourinary abnormalities. Condition is usually diagnosed prenatally by ultrasound visualization of enlarged kidney, with renal cortex being replaced by numerous cysts of multiple sizes with dysplastic parenchyma and absent calycle drainage system (Fig. 4). ${ }^{22}$ The parenchyma can be visualized in small islands between the cysts. Prognosis for unilateral disorder is good, while the bilateral condition, which is associated with decreased amniotic fluid level, is incompatible with life.

Solitary renal cyst is a rare condition in prenatal period and childhood. In the antenatal ultrasound assessment in the second trimester, the condition can be found with a frequency of 1 in 1,100 pregnancies $(0.09 \%) .{ }^{23}$ The diagnosis of a solitary renal cyst is made when there is a single, nonseptated cyst, with well-defined borders, without

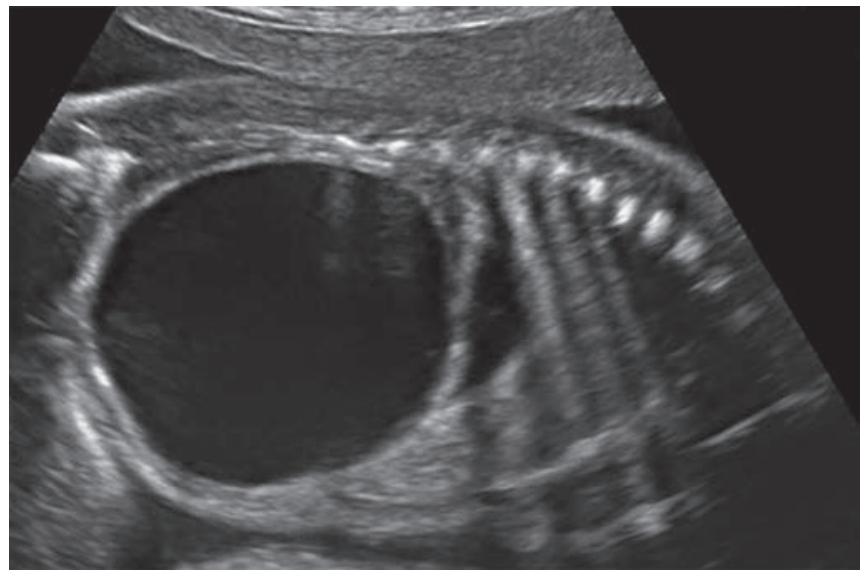

Fig. 5: Ultrasound examination in the second trimester revealing anechogenic solitary cyst in the projection of the left kidney

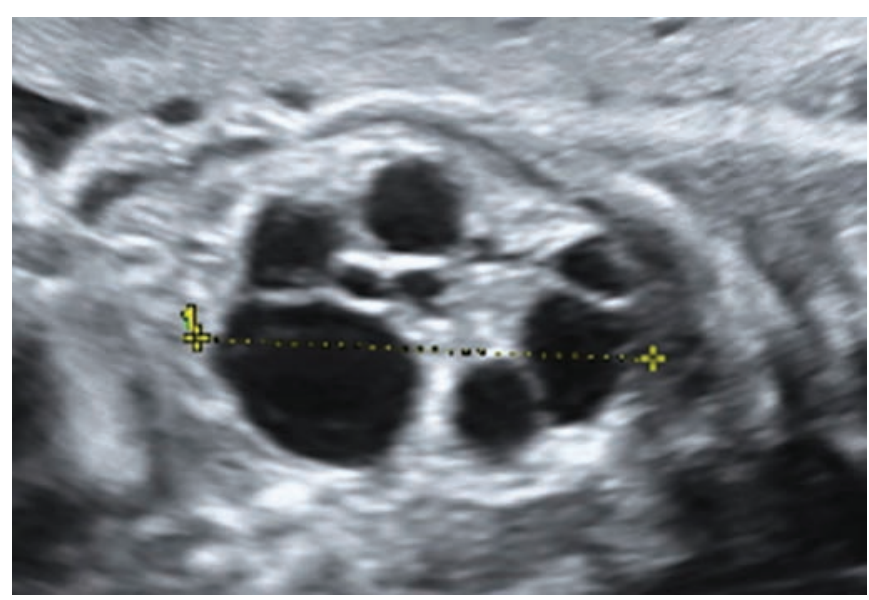

Fig. 4: Ultrasound assessment of enlarged kidney with many echogenic cysts of different sizes in the second trimester

communication between the cavity of the cyst and the renal collecting system, in an otherwise normal appearing kidney (Fig. 5). ${ }^{23}$ This condition is not usually associated with other anomalies. Solitary renal cyst has a transient nature and usually disappears by the end of the pregnancy or at early neonatal period, while persisting cysts do not increase in size and compromise renal function. ${ }^{23}$

Different renal abnormalities can be found in a several genetic syndromes, such as: Meckel-Gruber, Bardet-Biedel, and Beckwith-Wiedemann. Meckel-Gruber syndrome is a rare autosomal recessive lethal condition. The anomalies within the syndrome can be variable but the most common are dysplastic kidneys, olygohidramnios, occipital encephalocele, postaxial polydactyly, and limb shortening. Dysplastic kidneys consist of multiple microscopic cysts that destroy the tissue and increase the kidneys in size for more than 10 to 20 times, resulting in nonfunctional organs and decreased amount of amniotic fluid. Common ultrasound findings are enlarged kidneys containing cysts that increase in size toward the medulla with olygohidramnios (Fig. 6). The mean prevalence of

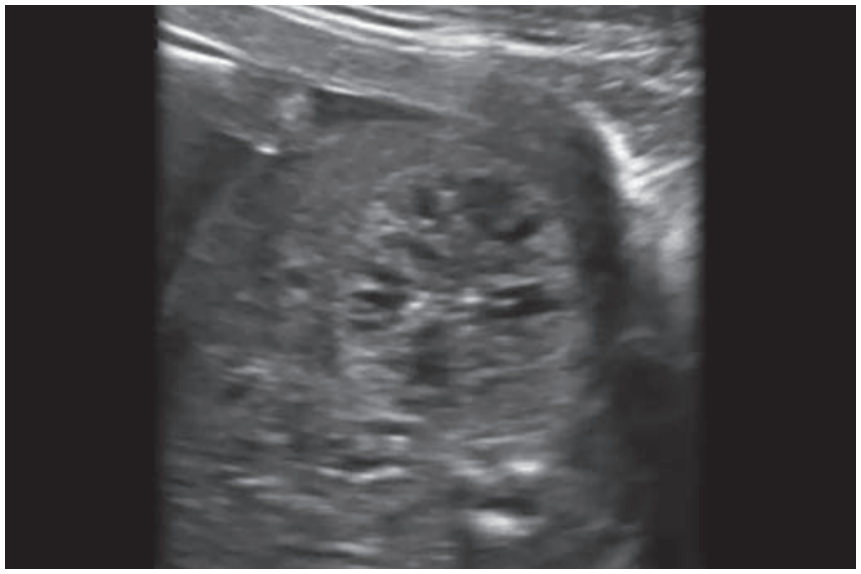

Fig. 6: Ultrasound finding of dysplastic kidneys in early second trimester with reduced amount of amniotic fluid in a case of MeckelGruber syndrome 


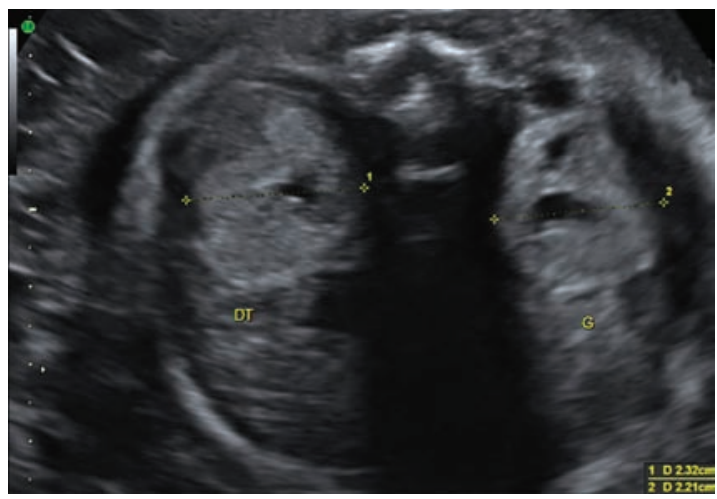

Fig. 7: Ultrasound finding of two enlarged and hyperechogenic kidneys with olygohidramnios in case of Bardet-Biedel syndrome

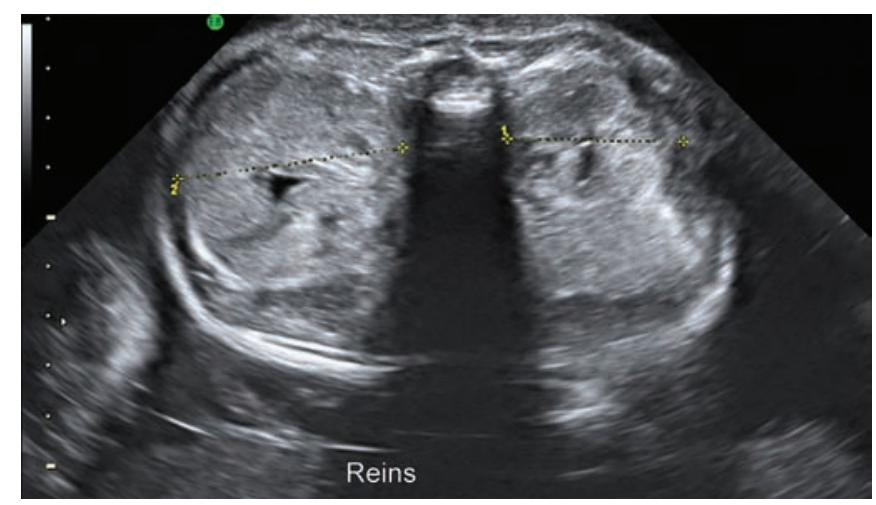

Fig. 8: Ultrasound examination of transversal section through fetal abdomen showed two kidneys increased in size with enhanced echodensity in a case of Beckwith-Wiedemann syndrome

organs with enhanced echogenicity accompanied by the normal or increased amount of the amniotic fluid (Fig. 8). Children with this syndrome are at higher risk to develop cancer during childhood, particularly Wilms' tumor (nephroblastoma), pancreatoblastoma, and hepatoblastoma, but do not have an increased risk of developing cancer in adulthood. In general, the prognosis is very good since most children with BeckwithWiedemann syndrome do not develop cancer and the vast majority of children who do develop cancer can be treated successfully.

Since most of the syndromes with renal involvement are autosomal recessive and relatively uncommon, the diagnosis could be hard to reach and recurrence may be difficult to predict. In any way, professional must try hard to provide the diagnosis due to its serious implications in the continuation or termination of pregnancy, evaluation of possible affection of other family members, and genetic counseling for the family. Although precise prenatal diagnosis of different kidney disease can be made using genetic testing of different fetal and chorionic materials, these methods are quite expensive and often unavailable in everyday practice so ultrasound evaluation remains the most widely used technique for diagnosis of different congenital kidney disorders. Although prenatal ultrasound assessment is an efficient method to examine renal anatomy, it has certain limitations in assessing renal function. Useful parameters can be amniotic fluid volume and echogenicity of renal parenchyma, both of which can be used as indicators of the underlying renal reserve. In better-equipped perinatal centers, ultrasound findings of different kidney anomalies can be followed by magnetic resonance imaging (MRI).

\section{ANOMALIES OF THE FETAL BLADDER}

By the end of the 7th gestational week the embryo has a cloaca, which is a single cavity at the caudal end of the in Beckwith-Wiedemann syndrome includes enlarged 
body. After that stage, the cloaca divides into two parts; ventral part that becomes the primary urogenital sinus and dorsal part that develops into the rectum. The urogenital sinus will further be subdivided into the cranial portion, which will form the future urinary bladder, while the caudal part will give the basis for future prostate, urethra, and external genitalia portions. Congenital anomalies of the lower urinary tract are a significant cause of neonatal morbidity and mortality in infancy so it is of great importance to diagnose these anomalies if possible in the antenatal period to provide the neonates timely set management and surgical treatment.

One of the most common congenital obstructive lesions of the urethra is posterior urethral valve that occurs only in phenotypic boys. This anomaly results from the formation of a thick membrane from the tissue of wolffian ducts. Posterior urethral valve can cause renal damage in children with end-stage renal disease in a subsequent proportion of children. The severity of the anomaly can be different depending on the various degrees of the obstruction within the urethra. ${ }^{27}$ Ultrasound manifestations of the posterior urethral valve can be different due to the severity of the anomaly. In most severe cases, fetus will be small for gestational age with oligohydramnios. The signs of the lower urinary tract indicative for posterior urethral valve are a keyhole sign, which is actually the combination of the bladder distention and upper part of the urethra immediately proximal to the valve together with the hypertrophy of the bladder wall. Additional findings can be hydronephrosis and hydroureter with different degrees of kidney dysplasia. It is important to stress out that all of these ultrasound features start to be visible from 26 weeks of the gestation onward, making hard to identify posterior urethral valve on routine detailed ultrasound examination usually carried out at 18 th week. ${ }^{28}$ Less severe cases are usually detected in postnatal period due to the repeated urinary infections. ${ }^{27}$

Cloacal malformations are rare congenital malformations that present only in phenotypic girls as remain of the structure from an early embryonic state. Cloacal malformations develop as a result of the disrupted division of the cloaca in the rectum and urogenital sinus, resulting in one common perineal opening for the urinary, genital, and gastrointestinal tracts, while the abdominal wall remains normal. ${ }^{29}$ Prenatal diagnosis based on the ultrasound can be hard to achieve due to the unexpected diagnosis and nonspecific ultrasound appearance of the anomaly. Most common sonographic findings are: Abdominal/pelvic cystic/mass, hydronephrosis, oligohydramnios, distended bowel/bowel obstruction, ascites, two-vessel cord, and dilated bladder. ${ }^{30}$ One should suspect the diagnosis of cloaca in a case of cystic abdominal masses with the combination of gastrointestinal and urological abnormalities in female fetus. Surgical management of the anomaly is pretty complicated, while the functional prognosis of individuals born with cloacal malformations with regard to achieving fecal and urinary continence is different depending on the complexity of the defect and the status of the spine and sacrum.

Bladder exstrophy is a rare congenital anomaly characterized by the protrusion of the urinary bladder through a defect in the abdominal wall. It can occur in both female and male fetuses with low incidence of about 1 in 10,000 to 1 in 50,000 of all pregnancies with higher incidence in females. ${ }^{31}$ This malformation is considered to be the result of cloacal division and is usually combined with the abnormalities of the bony pelvis, pelvic floor, and genitalia. Ultrasound features of urinary bladder exstrophy include normal amniotic fluid index with normal kidney appearance, while bladder could not be visualized. Additional findings are low insertion of the umbilical cord and defect of the anterior abdominal wall in the portion below the umbilicus. ${ }^{32}$ The prognosis for urinary continence is usually good after surgical correction but it depends upon other system anomalies.

\section{ANOMALIES OF THE FETAL GENITAL TRACT}

The most common anomaly of the fetal genital tract seen on ultrasound is ovarian cyst in female fetuses. It is also the most common intra-abdominal mass found by antenatal ultrasound in females. ${ }^{33} \mathrm{~A}$ fetal ovarian cyst can be of variable size and usually detected in the third trimester. It is believed that their occurrence is related with the action of different hormones of maternal and placental origin, such as estrogen and beta-human chorionic gonadotropin. Ultrasound appearance of the cyst is a well-circumscribed anechoic, unseptated cyst in the fetal pelvis separate from the fetal bladder, stomach, and gall bladder, which does not change its dimensions through pregnancy. ${ }^{33}$ These cysts are usually simple and uncomplicated with good prognosis and spontaneous resolution. Possible complications are rare, but include torsion, hemorrhage, and rupture.

Although external genital abnormalities are quite rare, the latest improvement of ultrasound technology, such as three- and four-dimensional high-definition (HD) ultrasound has enabled more precise assessment of genital organs and the correct establishment of the diagnosis in a case of malformations.

\section{CONCLUSION}

Ultrasound assessment through the pregnancy continues to be the most important source of clinical information in the evaluation of urogenital tract disorders. Additional 
molecular and genetic testing can be helpful in the process of diagnosis determination but are often unavailable and expensive with the lack of utility in a case of multifactorial disorders. Since fetal urogenital malformations are a significant cause of neonatal and child morbidity and mortality, the necessity of providing the correct diagnosis for the parents and professionals including in the management should be of great priority for the obstetricians dealing with ultrasound examination.

\section{REFERENCES}

1. Levi S. Ultrasound in prenatal diagnosis: polemics around routine ultrasound screening for second trimester fetal malformations. Prenat Diagn 2002 Apr;22(4):285-295.

2. Fadda GM, Capobianco G, Balata A, Litta P, Ambrosini G, D'Antona D, Cosmi E, Dessole S. Routine second trimester ultrasound screening for prenatal detection of fetal malformations in Sassari University Hospital, Italy: 23 years of experience in 42,256 pregnancies. Eur J Obstet Gynecol Reprod Biol 2009 Jun;144(2):110-114.

3. Van Dorsten JP, Hulsey TC, Newman RB, Menard MK. Fetal anomaly detection by second-trimester ultrasonography in a tertiary center. Am J Obstet Gynecol 1998 Apr;178(4):742-749.

4. Queisser-Luft A, Stolz G, Wiesel A, Schlaefer K, Spranger J. Malformations in newborn: results based on 30,940 infants and fetuses from the Mainz congenital birth defect monitoring system (1990-1998). Arch Gynecol Obstet 2002 Jul;266(3): 163-167.

5. Yosypiv IV. Congenital anomalies of the kidney and urinary tract: a genetic disorder? Int J Nephrol 2012;2012:909083.

6. Seikaly MG, Ho PL, Emmett L, Fine RN, Tejani A. Chronic renal insufficiency in children: the 2001 Annual Report of the NAPRTCS. Pediatr Nephrol 2003 Aug;18(8):796-804.

7. Available from: http://discovery.lifemapsc.com/library/ images/stages-in-kidney-development-pronephros-mesonephros-and-metanephros.

8. VlajkovicS, Dakovic-BjelakovicM,CukuranovicR,KrivokucaD. The average volume of fetal kidney during different periods of gestation. Acta Medica Medianae 2005;44(2):47-50.

9. Dias T, Sairam S, Kumarasiri S. Ultrasound diagnosis of fetal renal abnormalities. Best Pract Res Clin Obstet Gynaecol 2014 Apr;28(3):403-415.

10. Sairam S, Al-Habib A, Sasson S, Thilaganathan B. Natural history of fetal hydronephrosis diagnosed on mid-trimester ultrasound. Ultrasound Obstet Gynecol 2001 Mar;17(3):191-196.

11. Lee RS, Cendron M, Kinnamon DD, Nguyen HT. Antenatal hydronephrosis as a predictor of postnatal outcome: a metaanalysis. Pediatrics 2006 Aug;118(2):586-593.

12. Fernbach SK, Maizels M, Conway JJ. Ultrasound grading of hydronephrosis: introduction to the system used by the Society for Fetal Urology. Pediatr Radiol 1993;23(6):478-480.

13. Coco C, Jeanty P. Isolated fetal pyelectasis and chromosomal abnormalities. Am J Obstet Gynecol 2005 Sep;193(3 Pt 1): 732-738.

14. Torres VE, Harris PC, Pirson Y. Autosomal dominant polycystic kidney disease. Lancet 2007 Apr 14;369(9569):1287-1301.

15. Brun M, Maugey-Laulom B, Eurin D, Didier F, Avni EF. Prenatal sonographic patterns in autosomal dominant polycystic kidney disease: a multicenter study. Ultrasound Obstet Gynecol 2004 Jul;24(1):55-61.
16. Friedmann W, Vogel M, Dimer JS, Luttkus A, Büscher U, Dudenhausen JW. Perinatal differential diagnosis of cystic kidney disease and urinary tract obstruction: anatomic pathologic, ultrasonographic and genetic findings. Eur J Obstet Gynecol Reprod Biol 2000 Apr;89(2):127-133.

17. Sessa A, Righetti M, Battini G. Autosomal recessive and dominant polycystic kidney diseases. Minerva Urol Nefrol 2004 Dec;56(4):329-338.

18. Chaumoitre K, Brun M, Cassart M, Maugey-Laulom, Eurin D, Didier F, Avni EF. Differential diagnosis of fetal hyperechogenic cystic kidneys unrelated to renal tract anomalies: a multicentre study. Ultrasound Obstet Gynecol 2006 Dec;28(7):911-917.

19. Roume J, Ville Y. Prenatal diagnosis of genetic renal diseases: breaking the code. Ultrasound Obstet Gynecol 2004 Jul;24(1):10-18

20. Thomas J, Manjunath AP, Rai L, Kudva R. Autosomal recessive polycystic kidney disease diagnosed in fetus. Indian J Urol 2007 Jul-Sep;23(3):328-329.

21. Feldenberg LR, Siegel NJ. Clinical course and outcome for children with multicystic dysplastic kidneys. Pediatr Nephrol 2000 Oct;14(12):1098-1101.

22. Schreuder MF, Westland R, van WijkJA. Unilateral multicystic dysplastic kidney: a meta-analysis of observational studies on the incidence, associated urinary tract malformations and the contralateral kidney. Nephrol Dial Transplant 2009 Jun;24(6):1810-1818.

23. Blazer S, Zimmer E, Blumenfeld Z, Zelikovic I, Bronshtein M. Natural history of fetal simple renal cysts detected in early pregnancy. J Urol 1999 Sep;162(3 Pt 1):812-814.

24. Barisic I, Boban L, Loane M, Garne E, Wellesley D, Calzolari E, Dolk H, Addor MC, Bergman JE, Braz P, et al. Meckel-Gruber Syndrome: a population-based study on prevalence, prenatal diagnosis, clinical features, and survival in Europe. Eur J Hum Genet 2015 Jun;23(6):746-752.

25. Baker TM, Sturm EL, Turner CE, Petersen SM. Diagnosis of bardet-biedl syndrome in consecutive pregnancies affected with echogenic kidneys and polydactyly in a consanguineous couple. Case Rep Genet 2013;2013:159143.

26. Williams DH, Gauthier DW, Maizels M. Prenatal diagnosis of Beckwith-Wiedemann syndrome. Prenat Diagn 2005 Oct;25(10):879-884.

27. Nasir AA, Ameh EA, Abdur-Rahman LO, Adeniran JO, Abraham MK. Posterior urethral valve. World J Pediatr 2011 Aug;7(3):205-216.

28. Blews DE. Sonography of the neonatal genitourinary tract. Radiol Clin North Am 1999 Nov;37(6):1199-1208, vii.

29. Bischoff A, Levitt MA, Breech L, Louden E, Peña A. Hydrocolpos in cloacal malformations. J Pediatr Surg 2010 Jun;45(6):1241-1245.

30. Bischoff A, Levitt MA, Lim FY, Guimarães C, Peña A. Prenatal diagnosis of cloacal malformations. Pediatr Surg Int 2010 Nov;26(11):1071-1075.

31. Lattimer JK, Smith MJ. Exstrophy closure: a follow up on 70 cases. J Urol 1966 Mar;95(3):356-359.

32. Rudzinska M, Bijok J, Tomaszewska K, Massalska D, Gastol P, Ostrowska J, Jakiel G, Roszkowski T. Skin-covered bladder exstrophy diagnosed antenatally. J Ultrasound Med 2013 Nov;32(11):2043-2045.

33. Bryant AE, Laufer MR. Fetal ovarian cysts: incidence, diagnosis and management. J Reprod Med 2004 May;49(5):329-337. 